\title{
Visual assessment of dynamic knee joint alignment in patients with patellofemoral pain: an agreement study
}

\author{
Rudi Hansen ${ }^{\text {Corresp., } 1}$, Mathilde Lundgaard-Nielsen ${ }^{1}$, Marius Henriksen ${ }^{2}$ \\ ${ }^{1}$ Department of Physical and Occupational Therapy, Copenhagen University Hospital Bispebjerg and Frederiksberg, Copenhagen, Denmark \\ 2 The Parker Institute, Copenhagen University Hospital Bispebjerg and Frederiksberg, Copenhagen, Denmark \\ Corresponding Author: Rudi Hansen \\ Email address: jth299@alumni.ku.dk
}

Background. Assessment of knee kinematics plays an important role in the clinical examination of patients with patellofemoral pain (PFP). There is evidence that visual assessments are reliable in healthy subjects, but there is a lack of evidence in injured populations. The purpose of this study was to examine the intra- and interrater agreement in the visual assessment of dynamic knee joint alignment in patients with PFP.

Methods. The study was a cross-sectional agreement study. Sixty participants (42 females) were included. We assessed the intra- and interrater agreement of two functional tests: The single leg squat (SLS) and the forward lunge (FL). One investigator scored the movement according to preset criteria while video recording the movement for retest. Moreover, the performance was scored by another investigator using the video recording. Agreement was assessed using weighted kappa statistics.

Results. The intrarater agreement ranged from moderate to good (Kappa 0.58 (FL) to 0.70 (SLS)) whereas the interrater agreement ranged from fair to moderate (Kappa 0.22 (SLS) to 0.50 (FL)).

Conclusion. The agreement within raters was better than between raters, which suggests that assessments should preferably be performed by the same tester in research and in a clinical setting, e.g., to evaluate any treatment effect. We promote FL as a reliable clinical tool for evaluating dynamic knee alignment, since it shows equally good intra- and interrater agreement, and it is an inexpensive and easy method to use. 


\section{Visual assessment of dynamic knee joint alignment in 2 patients with patellofemoral pain: an agreement study}

3 Rudi Hansen ${ }^{1}$, Mathilde Lundgaard-Nielsen ${ }^{1}$, Marius Henriksen ${ }^{2}$

41 Department of Physical and Occupational Therapy, Copenhagen University Hospital

5 Bispebjerg and Frederiksberg, Copenhagen, Denmark

62 The Parker Institute, Copenhagen University Hospital Bispebjerg and Frederiksberg,

7 Copenhagen, Denmark

8

9 Corresponding Author:

10 Rudi Hansen ${ }^{1}$

11 Department of Physical and Occupational Therapy, Copenhagen University Hospital Bispebjerg

12 and Frederiksberg, Copenhagen, Denmark

13 Email address: rudi.hansen@regionh.dk 
38

39

40

41

42

43

44

45

46

47

48

49

50

51

52

53

54

55

56

57

58

59

60

61

62

63

64

65

66

67

68

69

70

71

72

73

74

75

76

77

\section{Abstract}

Background. Assessment of knee kinematics plays an important role in the clinical examination of patients with patellofemoral pain (PFP). There is evidence that visual assessments are reliable in healthy subjects, but there is a lack of evidence in injured populations. The purpose of this study was to examine the intra- and interrater agreement in the visual assessment of dynamic knee joint alignment in patients with PFP.

Methods. The study was a cross-sectional agreement study. Sixty participants (42 females) were included. We assessed the intra- and interrater agreement of two functional tests: The single leg squat (SLS) and the forward lunge (FL). One investigator scored the movement according to preset criteria while video recording the movement for retest. Moreover, the performance was scored by another investigator using the video recording. Agreement was assessed using weighted kappa statistics.

Results. The intrarater agreement ranged from moderate to good (Kappa 0.58 (FL) to 0.70

(SLS)) whereas the interrater agreement ranged from fair to moderate (Kappa 0.22 (SLS) to 0.50 $(\mathrm{FL})$ ).

Conclusion. The agreement within raters was better than between raters, which suggests that assessments should preferably be performed by the same tester in research and in a clinical setting, e.g., to evaluate any treatment effect. We promote FL as a reliable clinical tool for evaluating dynamic knee alignment, since it shows equally good intra- and interrater agreement, and it is an inexpensive and easy method to use.

\section{Background}

Malalignment of the lower extremity have been linked to musculoskeletal problems, including patellofemoral pain (PFP) (1-4). During movements such as squatting, individuals with PFP have demonstrated greater knee abduction excursion than controls $(5,6)$, and improvements in frontal and transverse plane pelvis and hip control have been linked to a reduction in pain (7). Therefore, physiotherapists use visual assessment of dynamic alignment in their clinical decision-making process when considering prescription of exercises (8).

Dynamic alignment can be measured by clinical observation or biomechanical motionanalysis technology. Development of three-dimensional biomechanical analyses has made it possible to quantify and evaluate knee kinematics during functional tasks in a valid and reliable manner (9). However, this method is generally costly and too time consuming in the clinical setting. Two-dimensional measures, such as the frontal plane projection angle and visual assessments or ratings of lower extremity motion, have been suggested as more cost effective and acceptable alternatives to three-dimensional motion capture $(6,10)$.

In visual assessments of frontal plane knee motion during a single leg squat (SLS) and a forward lunge (FL), the reliability is reported to range from moderate to excellent in nonsymptomatic subjects (10-15). Measurement properties of a test instrument are likely to depend on the population studied, and there is a lack of evidence in injured populations (16). In PFP patients reliability measures have been evaluated using a 2-D video capture procedure that 
78 quantifies frontal plane knee alignment during single limb squats (17). The results of this study

79 suggest that this method is reliable $(\mathrm{ICC}=0.86)$ in PFP patients. Measures of visual assessment

80 of lower extremity kinematics in PFP patients without the use of video analysis have been

81 limited to a single study evaluating a lateral step-down task (18). This study included the

82 evaluation of several aspects of movement quality (arm strategy, trunk movement, pelvis plane,

83 knee position and balance) and scored each item according to a scale designed for the study to

84 assess an overall movement quality. While this multimodal approach to evaluate movement

85 quality may be more comprehensive, it is difficult to compare the results to other more

86 commonly used measures of alignment and to extrapolate the findings to a clinical setting.

87

88

89

90

91

92

93

94

95

96

97

98

99

100

101

102

103

104

105

106

107

Because therapists use visual ratings to make clinical decisions, the reliability of these ratings needs to be considered. Our intention was to evaluate whether a simple visual rating of knee movement during two commonly used tests of dynamic alignment (the SLS and FL test) can be used reliably among PFP patients. The rating method used in this study resembles a clinical setting where the therapists do not have access to the equipment or time required for complex biomechanical analysis. The SLS and the FL were chosen because they are commonly used in clinical practice and have been reported in many previous studies investigating visual rating of lower extremity function (19). The tests are less demanding than the commonly used jump tests; they also more closely resemble activities of daily life, such as stair

ascending/descending, which may be more appropriate in a population of both sports-active and sedentary individuals.

The aims of this study were (a) to determine the intrarater agreement of a subjective visual assessment by an experienced sports physiotherapist in evaluating dynamic knee control during a SLS and a FL in a group of PFP patients, and (b) to determine the interrater agreement of the subjective assessment of dynamic knee control between two experienced sports physiotherapists.

\section{Materials \& Methods}

\section{Study design}

The study was a cross-sectional agreement study. The reporting of the study follows the

108

109 Guidelines for Reporting Reliability and Agreement Studies (GRRAS) (20).

110 Participants were a subset of an RCT study aiming to compare the effectiveness of therapeutic

111 hip and knee exercise for patients with PFP and to identify candidate patient characteristics that

112 predict differential responses to the two exercise programs (clinicaltrials.gov identifier:

113 NCT03069547). The study was pre-planned in the parent trial protocol in order to assess

114 measurement properties of the dynamic knee joint alignment measures, that will be assessed as a

115 potential patient characteristic associated with treatment response. The assessments were

116 performed at baseline in the parent trial, i.e., before randomization. As part of the baseline

117 information gathered in the parent trial the participants answered the Anterior Knee Pain Score (a 
118 specific disability score for PFP patients ranging from 0 to 100 points, where higher scores 119 indicate less disability (21)), and self-reported pain during the past 4 weeks assessed on a 0 ('no 120 pain') to 10 ('worst imaginable pain') Numeric Rating Scale. One participant failed to complete

121

122

123

124

125

126

127

128

129

130

131

132

133

134

135

136

137

138

139

140

141

142

143

144

145

146

147

148

149

150

151

152

153

154

155

156

157

the Anterior Knee Pain Score and 3 participants failed to answer one of the 13 questions. The reported mean is calculated for 59 participants and missing data handled by imputing the mean of the participants for that particular item as recommended by Hott et al. (22) and Chavance (23). Participants was recruited from the Institute of Sports Medicine Copenhagen (ISMC), Bispebjerg-Frederiksberg Hospital, Denmark. ISMC is a medical unit mainly for patients with injuries in the musculoskeletal system caused by participation in sports activities, and thus most participants are sports active. We aimed at including 60 participants in this sub-study, which gives $80 \%$ power to detect a kappa-coefficient of at least 0.5 that is statistically significantly different from 0 and corresponds to a moderate agreement.

\section{Raters}

Raters were RH (male) and MLN (female) who are both sports physiotherapists with 18 and 15 years of experience, respectively, in treating and assessing patients with musculoskeletal problems. Both raters use visual assessments as part of their daily clinical practice but have not used it for research purposes.

\section{General procedures}

Video was recorded using a tablet (Apple Ipad Air 2, frame rate: 30 frames per second) from an anterior view of participants performing the SLS and FL in the gym at the Department of Physical and Occupational Therapy at Bispebjerg and Frederiksberg Hospital. An investigator (RH) instructed the participants to perform the selected movement as described below. After the instruction had been understood, the participant performed the selected movement without rehearsal. If the participant lost his/her balance during the test, a new trial was performed. No discussion of the testing procedures or the classification criteria occurred during the testing. The investigator filmed the participant and simultaneously scored the movement as observed clinically according to the criteria set below. The tablet was set up directly in front of participants, perpendicular to the frontal plane at a height of $100 \mathrm{~cm}$ and a distance of $3.5 \mathrm{~m}$. The video captured the whole person. At least 1 week later, the investigator did another scoring based on the recorded video and another investigator (MLN) repeated the scoring independently. Three playbacks of the recorded video in real time were allowed for the intra- and interrater assessment.

\section{Knee alignment during single leg squat}

The SLS test has been described in several studies and the present procedure was a replication of comparable agreement studies $(10,13,24)$. From a position of single leg standing (painful knee), individuals performed a partial squat on one leg (hip and knee flexion) with the trunk maintained in an upright position, the contralateral hip in neutral and contralateral knee flexed. Individuals 
158 were instructed to perform the squat until they reached maximum ankle dorsiflexion without 159 lifting their heels and then return to upright standing (Figure 1a). The SLS was performed at 160 participant-selected speed.

161

\section{Knee alignment during forward lunge}

163

The FL test was performed according to comparable agreement studies $(24,25)$. From a position

164

165

166

167

168

169

170

171

172

173

174

175

176

177

178

179

180

181

182

183

184

185

186

187

188

189

190

191

192

193

194

195

196

197 of bilateral stance, individuals performed a forward step (painful knee), and continued the motion by flexing the front and back knee simultaneously (forward lunge).

Individuals were instructed to continue the lunge until reaching maximum dorsiflexion of the stance leg without lifting their heel and to push-off to upright position (Figure 1b). The FL was performed at participant-selected speed. For both tests, dynamic valgus alignment was defined as an excessive medial movement of the knee as evidenced by an apparent increased frontal plane knee angle during the selected movement. Varus alignment was defined as an excessive lateral movement.

\section{Scoring}

The rater determined if a dynamic worsening of valgus or varus was present and scored the movement using the following categories:

$-4=$ severe valgus

$-3=$ moderate valgus

$-2=$ mild valgus

$-1=$ doubtful valgus

$0=$ no evidence of neither valgus nor varus

$1=$ doubtful varus

$2=$ mild varus

$3=$ moderate varus

$4=$ severe varus

We defined 'no evidence of neither valgus nor varus' as a neutral knee alignment, i.e. knee flexion aligned with the $2^{\text {nd }}$ toe. We considered 'doubtful' a just merely detectable deviation from neutral alignment, while 'mild', 'moderate' and 'severe' was considered a definite deviation from neutral. The raters ranked the deviations 'mild', 'moderate' or 'severe' based on the experience of the raters in assessing PFP patients. We defined 'mild' as a slight deviation that might not be clinically relevant, 'moderate' as a modest and clinically relevant deviation from neutral, and 'severe' as a clinically relevant and severe collapse of the knee.

Similar ordinal and nominal scales have previously been used in the assessment of intra- and interrater agreement (11,26-29). Visual assessments of dynamic knee joint alignment have been validated against a 'gold standard', i.e. three-dimensional motion analysis, and the ability of visual assessments to identify 'true' malalignment is considered acceptable $(13,19)$. 
198

199

200

201

202

203

204

205

206

207

208

209

210

211

212

213

214

215

216

217

218

219

220

221

222

223

224

225

226

227

228

229

230

231

232

233

234

235

236

237

\section{Single leg squat and forward lunge classifications:}

An a priori categorical classification was made where the scores -4 to -2 were categorized as 'Definite valgus present', the scores $-1,0$ and 1 were categorized as 'No definite evidence of dynamic malalignment' and the scores 2 to 4 were categorized as 'Definite varus present'. Conversion of scores into categorical variables is recommended in order to simplify the ratings (19).

\section{Statistical methods}

Statistical analysis was completed using SAS (version 9.1 for Windows; SAS Institute Inc, Cary, NC). Descriptive statistics were calculated for demographics. Weighted kappa values with 95\% CIs were used to examine the intratester and intertester reliability of the visual assessment. Cohens weighted kappa values with $95 \%$ CIs were used to examine the intratester and intertester reliability of the visual assessment. Cohens weighted kappa is broadly used and is a robust statistic useful for interrater and intrarater reliability testing (30). Agreement was assessed using the classification for each movement test and for raw data (scores from -4 to 4 ). Interpretations of Kappa values were based on the guidelines adapted from Landis and Koch (31): $<0.20$ : Poor agreement; 0.21-0.40: Fair agreement; 0.41-0.60: Moderate agreement; 0.61-0.80: Good agreement; 0.81-1.00: Very good agreement. We aimed at including 60 participants which gave $80 \%$ power to detect a kappa-coefficient of at least 0.5 , which corresponds to moderate agreement.

\section{Ethical Considerations}

Ethics approval was given by the Health Research Ethics Committee of the Capital Region of Denmark (De Videnskabsetiske Komitéer for Region Hovedstaden), protocol \#H-16045755. Participants written informed consent were obtained prior to the start of the study.

\section{Results}

The first sixty individuals with PFP who were included in the parent trial accepted the invitation to participate in this agreement study. Their characteristics are shown in table 1 . The dispersion of the data from the intra- and interrater assessments are presented in a heat map in figure 2 (SLS) and figure 3 (FL). The weighted kappa values for the classifications and the raw scores are shown in tables 2 and 3 . In summary, the intrarater agreement were statistically significantly different from $0(\mathrm{p}<0.0001)$ and ranged from 0.58 to 0.70 , i.e., moderate to good agreement, whereas the interrater agreement ranged from $0.22(p=0.08)$ to $0.50(p<0.0001)$, i.e. fair to moderate agreement. Interrater agreement was generally not as good as intrarater agreement $(0.7$ for SLS intrarater classification scores vs. 0.22 for interrater scores, and 0.58 for FL intrarater classification scores vs. 0.48 for interrater scores). The mean time from baseline to re-evaluation in the intrarater assessment was 29.1 days (SD 14.8). The cross tabulated agreements in the classifications and raw scores are provided in the supplementary file. 


\section{Discussion}

\section{Principal findings}

241 The aim of this study was to determine the intra- and interrater agreement between two

242 experienced physiotherapists when visually assessing the dynamic knee alignment during an SLS

243 and an FL in a population of PFP patients. The most important finding was that the visual

244 assessments of dynamic alignment during SLS and FL can be done reliably when the assessment

245 is repeated by the same rater. Moreover, 'moderate' levels of agreements are seen when two

246 experienced raters assess the FL, while the interrater agreement is only 'fair' when assessing the

247 SLS.

248

\section{Comparison with previous studies}

250

251

The intrarater agreements for the SLS in the current study compare to the results of a review on

252 the interrater and intrarater agreement of observation-based assessment of the SLS including studies on both healthy subjects and subjects with lower extremity disorders (32). In that study, the pooled results of Kappa showed a 'substantial' agreement for intrarater agreement (Kappa

254 value 0.68 (95\% CI 0.60 to 0.74$)$ ). Moreover, the review found a 'moderate' agreement for interrater reliability of the SLS (Kappa value 0.58 (95\% CI 0.50 to 0.65 )), which is somewhat higher than in present study. In the present study no efforts were made to synchronize assessors by mutual training sessions or by operationalizing the measurements. Visual assessments were therefore entirely based on the experience of the assessors. This might explain the discrepancy with the systematic review and the relatively low interrater agreements seen.

260

261

\section{Clinical implications}

262 The clinical implications of the results of our study are, that visual assessment of frontal plane 263 knee kinematics during a FL can be done reliably by experienced testers, whereas SLS should 264 preferably be re-evaluated by the same tester. Forward lunge is therefore a reliable clinical tool 265 for evaluating knee alignment. Furthermore, it is an inexpensive and easy method to use, making 266 it ideal for the clinical setting. However, the clinical validity, relevance, and prognostic value 267 still need to be established.

\section{Strengths and limitations}

This study has some strengths and limitations. Firstly, by using an objective measurement tool

272 verbal or non-verbal interaction between raters. Using the tablet, on the other hand, implies an

273 inherent limitation by not taking the variability of the patients' performances into account. It was

274 out of the scope of this study to include a clinical re-evaluation of the participants and we

275 thereby accept the exclusion of data on within subject variability. On the other hand, this enables

276 a focused analysis of the agreement within and between raters. 
277

278

279

280

281

282

283

284

285

286

287

288

289

290

291

292

293

294

295

296

297

298

299

300

301

302

303

304

305

306

307

308

309

310

311

312

313

314

315

316

Another strength is, that we have included a relatively large sample of PFP patients, which makes the results transferable to other clinical and experimental settings. It should be noted, though, that assessors were experienced, and our results may only be transferable when assessments are made by equally experienced assessors.

In our assessment of movement quality, we only included the knee excursion during the movement (dynamic knee valgus or varus). Rating dynamic knee alignment per se is not an exhaustive evaluation of movement quality and is merely one aspect of the full range clinical examinations. It is, however, considered a good indicator of movement quality (33) with a kneemedial-to-foot position often considered less optimal, indicating poor postural orientation (13, 34). A limitation of assessing movement quality without three-dimensional analyses is, that we lack information on movement components like transversal and sagittal plane control of body segments. However, we aimed solely at assessing the reliability of the test assessments and not on the validity of the actual assessments. Furthermore, we did not control for the speed or acceleration of the movement. We intentionally omitted the control in order to comply with our intents of resembling a clinical setup. We acknowledge the limitation of not including the control of speed and acceleration of the execution of the exercises and that speed and acceleration may have influenced the knee excursion.

Various scoring systems have been used to assess dynamic knee joint alignment in the literature (32). The scoring system used in this study resembles previous used systems $(26,29)$ Chmielewski et al. (26) used the terms "no deviation from neutral alignment", "small-magnitude or barely observable movement out of a neutral position", "moderate-magnitude or marked movement out of a neutral position, and "excessive or severe magnitude of movement out of a neutral position", to assess the degree of knee excursion during a unilateral squat and lateral stepdown task. We made the scoring two-tailed (varus and valgus) to be more specific in the direction of the knee in the movement. The use of different scoring systems in the literature, makes it difficult to compare and pool the results of agreement studies. Future studies should consider standardizing the scoring for the benefit of research in reliability and agreement of knee kinematics.

No varus knees ( $>1$ on the scoring scale) were found in the population. This means that when scores were converted into classifications there were only two viable options so it is difficult to tell if the results would be similar if individuals with varus were included in the analysis. The narrow range of scores is probably linked to the population, indicating that PFP patients are more prone to a valgus knee alignment.

Comparing real time scoring to retrospective scoring may have impacted our results. We chose the real time visual assessment in order to resemble a clinical setup and accepted the potential bias of re-evaluating the movement on a screen. We argue, however, that since the assessment was only in the frontal plane, the risk of bias in the subsequent re-rating on a screen was small. Further, the re-assessment of the video recordings did not include slow motion, which makes the validity of the results representative of clinical practice. 


\section{Conclusions}

318 Visual assessments of dynamic knee joint alignment during a FL and a SLS performed by

319

320

321

322

323

324

325

326

327

328

329

330

331

332

333

334

335

336

337

338

339

340

341

342

343

344

345

346

347

348

349

350

351

352

353

354

355

356

357

358

359

patients with PFP can be done reliably when an experienced rater repeats the assessment. Two experienced physiotherapists agree moderately when assessing dynamic alignment during FL, but only 'fair' when assessing dynamic alignment during SLS. The agreement within raters is better than between raters, which suggests that assessments should preferably be performed by the same tester in research and in a clinical setting, e.g., to evaluate any treatment effect. We suggest the FL as a reliable clinical tool for evaluating knee alignment in the clinical setting, since it shows acceptable intra- and interrater agreement, and it is an inexpensive and easy test to use.

\section{References}

1. Myer GD, Ford KR, Di Stasi SL, Foss KD, Micheli LJ, Hewett TE. High knee abduction moments are common risk factors for patellofemoral pain (PFP) and anterior cruciate ligament (ACL) injury in girls: is PFP itself a predictor for subsequent ACL injury? Br J Sports Med. 2015;49(2):118-22.

2. Willson JD, Binder-Macleod S, Davis IS. Lower extremity jumping mechanics of female athletes with and without patellofemoral pain before and after exertion. Am J Sports Med. 2008;36(8):1587-96.

3. Powers CM. The influence of altered lower-extremity kinematics on patellofemoral joint dysfunction: a theoretical perspective. J Orthop Sports Phys Ther. 2003;33(11):639-46.

4. Gwynne CR, Curran SA. Two-dimensional frontal plane projection angle can identify subgroups of patellofemoral pain patients who demonstrate dynamic knee valgus. Clin Biomech (Bristol, Avon). 2018;58:44-8.

5. Nakagawa TH, Moriya ET, Maciel CD, Serrao FV. Trunk, pelvis, hip, and knee kinematics, hip strength, and gluteal muscle activation during a single-leg squat in males and females with and without patellofemoral pain syndrome. J Orthop Sports Phys Ther. 2012;42(6):491-501.

6. Willson JD, Davis IS. Utility of the frontal plane projection angle in females with patellofemoral pain. J Orthop Sports Phys Ther. 2008;38(10):606-15.

7. Mascal CL, Landel R, Powers C. Management of patellofemoral pain targeting hip, pelvis, and trunk muscle function: 2 case reports. J Orthop Sports Phys Ther. 2003;33(11):64760.

8. Stephen J, Ephgrave C, Ball S, Church S. Current concepts in the management of patellofemoral pain - The role of alignment. Knee. 2020;27(2):280-6.

9. Mok KM, Petushek E, Krosshaug T. Reliability of knee biomechanics during a vertical drop jump in elite female athletes. Gait Posture. 2016;46:173-8.

10. Harris-Hayes M, Steger-May K, Koh C, Royer NK, Graci V, Salsich GB. Classification of lower extremity movement patterns based on visual assessment: reliability and correlation with 2-dimensional video analysis. J Athl Train. 2014;49(3):304-10.

11. Weeks BK, Carty CP, Horan SA. Kinematic predictors of single-leg squat performance: a comparison of experienced physiotherapists and student physiotherapists. BMC Musculoskelet Disord. 2012;13:207. 
360 12. Stensrud S, Myklebust G, Kristianslund E, Bahr R, Krosshaug T. Correlation between 361 two-dimensional video analysis and subjective assessment in evaluating knee control among elite 362 female team handball players. Br J Sports Med. 2011;45(7):589-95.

363 13. Ageberg E, Bennell KL, Hunt MA, Simic M, Roos EM, Creaby MW. Validity and inter364 rater reliability of medio-lateral knee motion observed during a single-limb mini squat. BMC 365 Musculoskelet Disord. 2010;11:265.

366 14. Jones D, Tillman SM, Tofte K, Mizner RL, Greenberg S, Moser MW, et al.

367 Observational ratings of frontal plane knee position are related to the frontal plane projection 368 angle but not the knee abduction angle during a step-down task. J Orthop Sports Phys Ther. 369 2014;44(12):973-8.

370 15. Tate J, True H, Dale B, Baker C. Expert versus Novice Interrater and Intrarater 371 Reliability of the Frontal Plane Projection Angle during a Single-Leg Squat. International 372 Journal of Athletic Therapy and Training. 2015;20(4):23-7.

373 16. Whatman C, Toomey C, Emery C. Visual rating of movement quality in individuals with 374 and without a history of intra-articular knee injury. Physiother Theory Pract. 2019:1-7.

375 17. Gwynne CR, Curran SA. Quantifying frontal plane knee motion during single limb 376 squats: reliability and validity of 2-dimensional measures. Int J Sports Phys Ther. 2014;9(7):898377906.

378 18. Piva SR, Fitzgerald K, Irrgang JJ, Jones S, Hando BR, Browder DA, et al. Reliability of 379 measures of impairments associated with patellofemoral pain syndrome. BMC Musculoskelet 380 Disord. 2006;7:33.

381 19. Whatman C, Hume P, Hing W. The reliability and validity of physiotherapist visual 382 rating of dynamic pelvis and knee alignment in young athletes. Phys Ther Sport. 2013;14(3):16838374.

384 20. Kottner J, Audige L, Brorson S, Donner A, Gajewski BJ, Hrobjartsson A, et al.

385 Guidelines for Reporting Reliability and Agreement Studies (GRRAS) were proposed. Int J Nurs 386 Stud. 2011;48(6):661-71.

387 21. Kujala UM, Jaakkola LH, Koskinen SK, Taimela S, Hurme M, Nelimarkka O. Scoring of 388 patellofemoral disorders. Arthroscopy. 1993;9(2):159-63.

389 22. Hott A, Liavaag S, Juel NG, Brox JI, Ekeberg OM. The reliability, validity, 390 interpretability, and responsiveness of the Norwegian version of the Anterior Knee Pain Scale in 391 patellofemoral pain. Disabil Rehabil. 2021;43(11):1605-14.

392 23. Chavance M. Handling Missing Items in Quality of Life Studies. Communications in 393 Statistics - Theory and Methods. 2004;33(6):1371-83.

394 24. Nae J, Creaby MW, Nilsson G, Crossley KM, Ageberg E. Measurement Properties of a 395 Test Battery to Assess Postural Orientation During Functional Tasks in Patients Undergoing 396 Anterior Cruciate Ligament Injury Rehabilitation. J Orthop Sports Phys Ther. 2017;47(11):86339773.

398 25. Alkjaer T, Simonsen EB, Peter Magnusson SP, Aagaard H, Dyhre-Poulsen P. Differences 399 in the movement pattern of a forward lunge in two types of anterior cruciate ligament deficient 400 patients: copers and non-copers. Clin Biomech (Bristol, Avon). 2002;17(8):586-93.

401 26. Chmielewski TL, Hodges MJ, Horodyski M, Bishop MD, Conrad BP, Tillman SM. 402 Investigation of clinician agreement in evaluating movement quality during unilateral lower 403 extremity functional tasks: a comparison of 2 rating methods. J Orthop Sports Phys Ther. 404 2007;37(3):122-9. 
405 27. Junge T, Balsnes S, Runge L, Juul-Kristensen B, Wedderkopp N. Single leg mini squat: 406 an inter-tester reproducibility study of children in the age of 9-10 and 12-14 years presented by 407 various methods of kappa calculation. BMC Musculoskelet Disord. 2012;13:203.

408 28. Trulsson A, Garwicz M, Ageberg E. Postural orientation in subjects with anterior cruciate 409 ligament injury: development and first evaluation of a new observational test battery. Knee Surg 410 Sports Traumatol Arthrosc. 2010;18(6):814-23.

411 29. Whatman C, Hing W, Hume P. Physiotherapist agreement when visually rating 412 movement quality during lower extremity functional screening tests. Phys Ther Sport. 413 2012;13(2):87-96.

414 30. McHugh ML. Interrater reliability: the kappa statistic. Biochem Med (Zagreb). $415 \quad 2012 ; 22(3): 276-82$.

416 31. Landis JR, Koch GG. The measurement of observer agreement for categorical data. 417 Biometrics. 1977;33(1):159-74.

418 32. Ressman J, Grooten WJA, Rasmussen Barr E. Visual assessment of movement quality in 419 the single leg squat test: a review and meta-analysis of inter-rater and intrarater reliability. BMJ 420 Open Sport Exerc Med. 2019;5(1):e000541.

421 33. Sahrmann S, Azevedo DC, Dillen LV. Diagnosis and treatment of movement system 422 impairment syndromes. Braz J Phys Ther. 2017;21(6):391-9.

423 34. Ortqvist M, Mostrom EB, Roos EM, Lundell P, Janarv PM, Werner S, et al. Reliability 424 and reference values of two clinical measurements of dynamic and static knee position in healthy 425 children. Knee Surg Sports Traumatol Arthrosc. 2011;19(12):2060-6. 
Figure 1

Screenshots of video recordings in the assessment of a single leg squat (a) and a forward lunge (b)

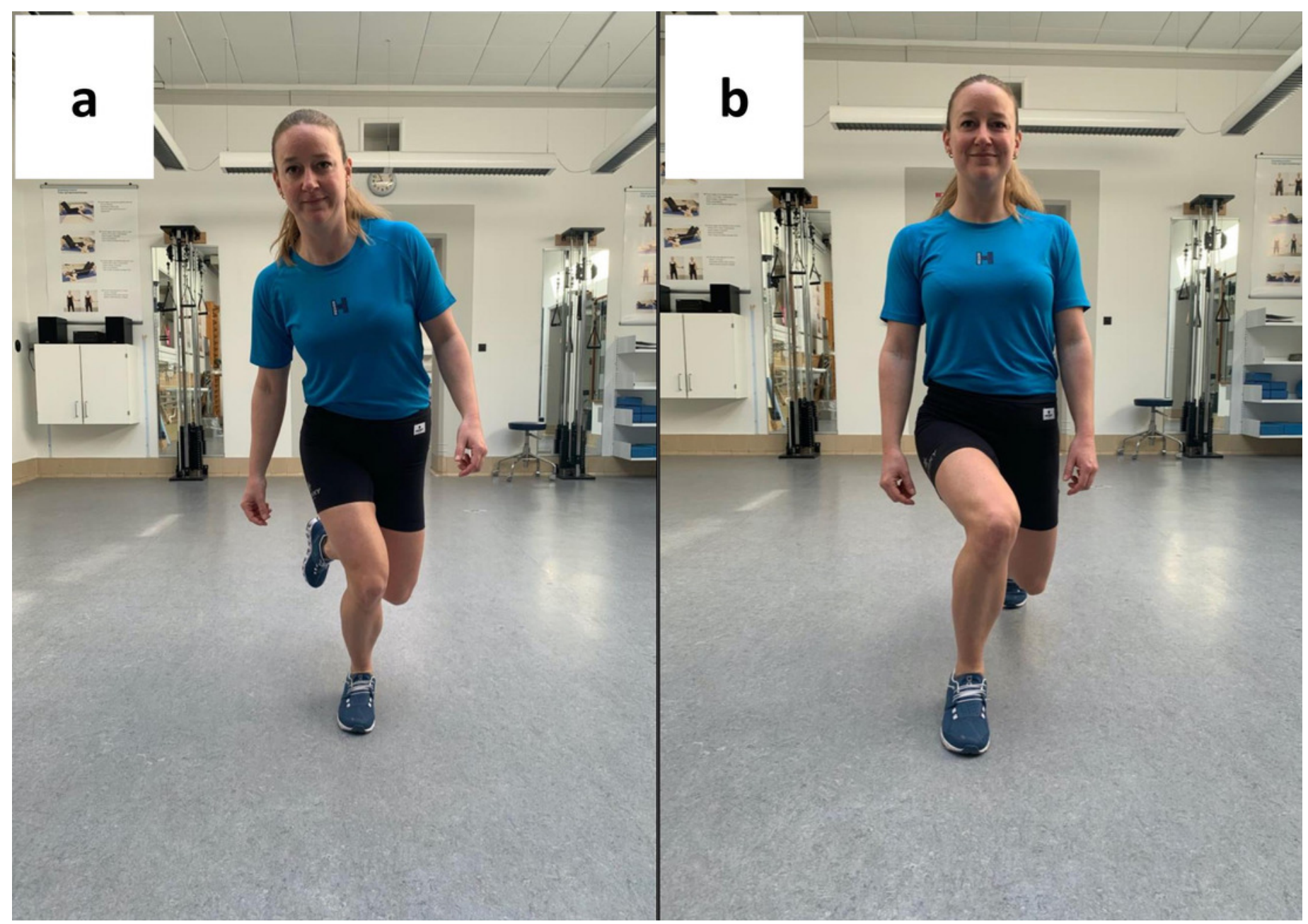


Figure 2

Heatmap of agreement matrix showing the dispersion of the intrarater (left) and interrater agreement (right) for the single leg squat.

The brightness of the blue color indicates the number of rating combinations with darker colors representing higher numbers as shown in the individual squares and in the key bar.
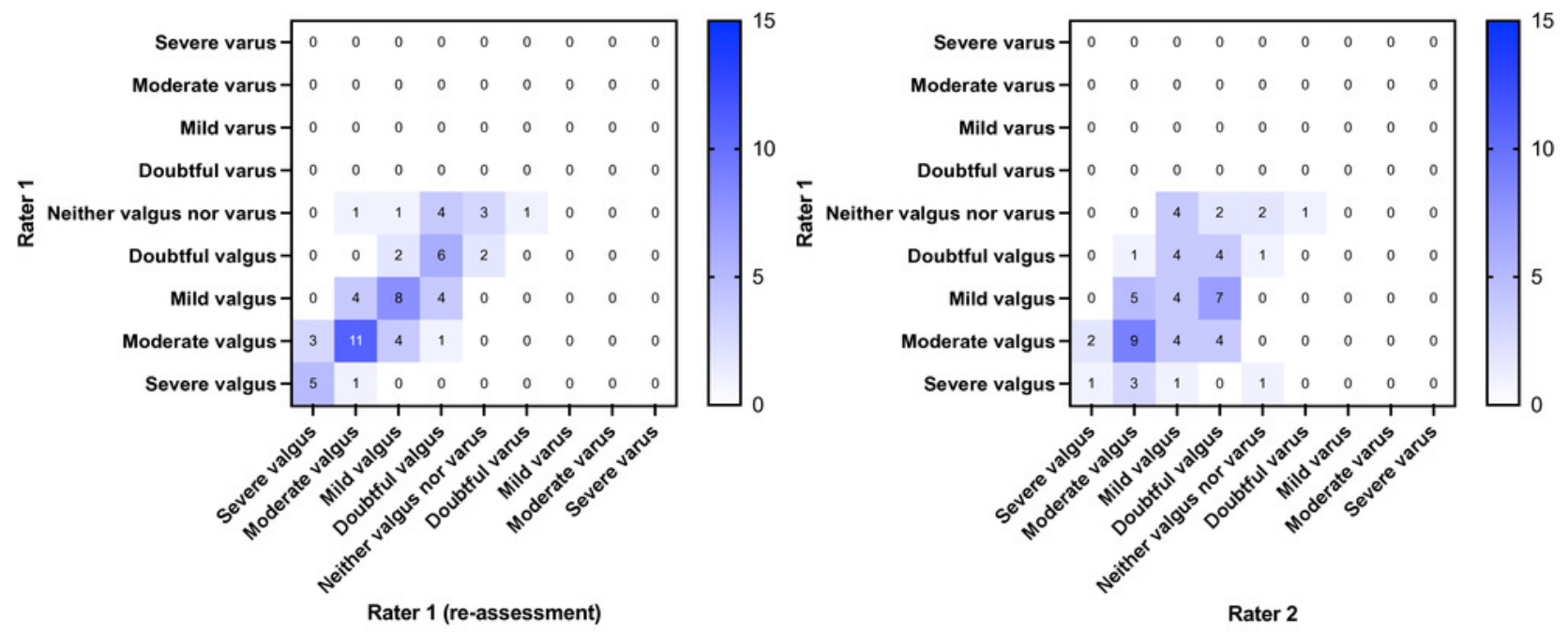
Figure 3

Heatmap of agreement matrix showing the dispersion of the intrarater (left) and interrater agreement (right) for the forward lunge.

The brightness of the blue color indicates the number of rating combinations with darker colors representing higher numbers as shown in the individual squares and in the key bar.
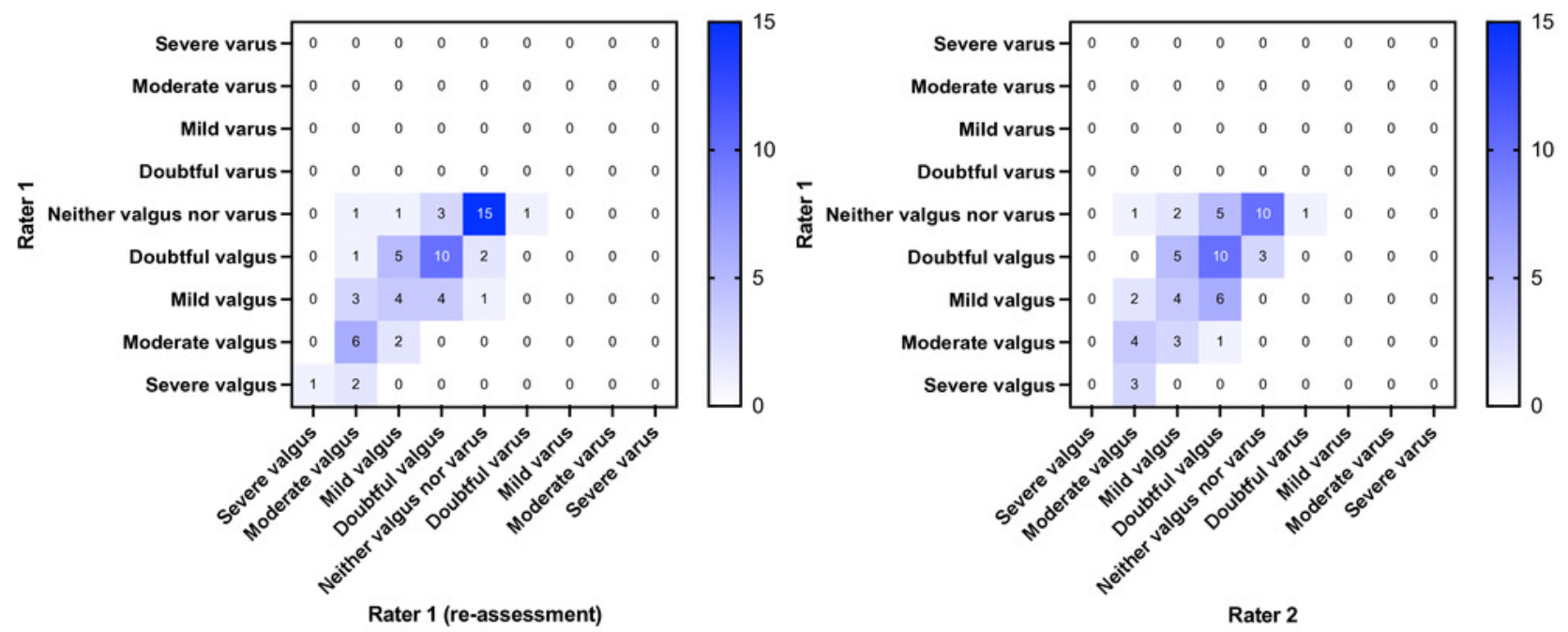
Table $\mathbf{1}$ (on next page)

Descriptive characteristics of the participants $(n=60)$ 


\begin{tabular}{lc}
\hline Characteristics & Mean (SD) \\
\hline Age (yrs) & $27.2(6.2)$ \\
Females (n (\%)) & $42(70 \%)$ \\
Height (cm) & $172.1(8.6)$ \\
Weight (kg) & $66.6(10.6)$ \\
Body mass index & $22.4(2.8)$ \\
Duration of symptoms (months) & $34(34)$ \\
Anterior Knee Pain Score (0-100 points) & $30.20(5.15)$ \\
Average pain during previous 4 weeks (NRS* 0-10) & $3.73(2.17)$ \\
\hline *Numeric Rating Scale &
\end{tabular}

1 
Table 2 (on next page)

Intra- and interrater agreement of single leg squat 
1

\begin{tabular}{llll}
\hline & $\begin{array}{l}\text { Weighted } \\
\text { kappa value }\end{array}$ & $95 \%$ CI & p-value \\
\hline Intrarater classification & 0.70 & $0.51-0.89$ & $<.0001$ \\
Intrarater raw data & 0.65 & $0.54-0.76$ & $<.0001$ \\
Interrater classification & 0.22 & $-0.03-0.48$ & 0.08 \\
Interrater raw data & 0.32 & $0.16-0.48$ & 0.05 \\
\hline
\end{tabular}


Table 3 (on next page)

Intra- and interrater agreement of forward lunge 
1

\begin{tabular}{llll}
\hline & $\begin{array}{l}\text { Weighted } \\
\text { kappa value }\end{array}$ & $95 \%$ CI & p-value \\
\hline Intrarater classification & 0.58 & $0.37-0.79$ & $<.0001$ \\
Intrarater raw data & 0.65 & $0.53-0.78$ & $<.0001$ \\
Interrater classification & 0.48 & $0.25-0.7$ & 0.0002 \\
Interrater raw data & 0.50 & $0.36-0.64$ & $<.0001$ \\
\hline
\end{tabular}

